\title{
AC 2008-409: REMOTE NONDESTRUCTIVE TESTING EDUCATIONAL LABORATORY
}

\section{Vladimir Genis, Drexel University}

Dr. Vladimir Genis, Associate Professor and Program Director of Applied Engineering

Technology in the Goodwin College, Drexel University, taught and developed graduate and undergraduate courses in physics, electronics, nondestructive testing, biomedical engineering, and acoustics. His research interests include ultrasound wave propagation and scattering, ultrasound imaging, nondestructive testing, electronic instrumentation, piezoelectric transducers, and engineering education. He serves as a member of the Drexel's Faculty Senate.

\section{Michael Zagorski, Drexel University}

Michael Zagorski has a wide range of academia, research and industrial experience in the areas of electronics, telecom/wireless, aerospace, and medical devices. He works as a product development engineer at Rheomedix, Inc. where he is involved in all aspects of new product development of medical devices. He graduated from Drexel University with a BS degree in Electrical Engineering in 2003 and MS degree in Biomedical Engineering in 2005. Currently, he is pursuing a Ph. D. degree in Biomedical Engineering. 


\title{
Remote Nondestructive Testing Educational Laboratory
}

\begin{abstract}
Drexel University's remote Internet-based nondestructive testing (NDT) laboratory for engineering technology students is described in this paper. Drexel's Goodwin College of Professional Studies submitted a proposal entitled "Implementation of the Internet-Based Nondestructive Evaluation Laboratory for Applied Engineering Technology Curriculum" as a reply to NSF solicitation under the program Course, Curriculum, Laboratory Implementation (CCLI) in May 2006. According to the proposal, Drexel would adapt Iowa State University's instructional material to its own ultrasound imaging laboratory course by placing emphasis on NDT techniques and applications. Once fully developed, the NDT laboratory would serve as a training center for Applied Engineering Technology (AET) students, as well as for employees of the companies involved in NDT. The proposal received a very favorable response from the reviewers, and the NSF panel and was considered for funding. Nevertheless, the NSF panel addressed some questions that came up during the review process. After receiving PI and co-PIs' responses, the project was awarded. The laboratory procedures were developed and set-up and the course was offered to pre-junior AET students. The videoconference-based instruction of the course is under development. The Internet-based videoconferencing mode will allow students at community colleges partnering with Drexel University participation in laboratory activities remotely.
\end{abstract}

\section{INTRODUCTION}

Nondestructive testing (NDT) is one of the most powerful and cost-effective techniques for quality and safety control of structures, parts, and products. NDT of materials and components is crucial to aerospace, naval, railroad, and other industries. For example, aerospace designers and manufacturers are frequently faced with the need to validate the integrity of structural parts for military and commercial aircrafts. Transportation equipment is highly specialized and safety sensitive therefore, it is important that all aspects of a failure are investigated, not only for classifying the failure mode but also determining its cause from an engineering mechanics and design point of view. Similar issues exist in other areas, such as maintenance and diagnostic techniques for nuclear power plants and petrochemical industry. The proposed project was designed in response to the requirements of local and regional industries in need of such techniques, and more importantly, of qualified personnel. Due to its distinctive nature, Drexel's Applied Engineering Technology program is uniquely positioned to incorporate NDT courses and experiences as part of its curriculum, thus creating the necessary knowledge and experience among its students and graduates.

To address these needs, we have developed the following objectives for this project:

- To provide students with hands-on, project- or laboratory-based courses in AET. 
- To stimulate students' interest in further study in AET.

- To introduce students to concepts, tools, and techniques utilized in real-world industrial applications.

An introductory, laboratory-based course in ultrasound imaging was developed and offered at the undergraduate level in the School of Biomedical Engineering at Drexel University. ${ }^{1}$ Lecture time with students allowed for the introduction of topics included in the laboratory experience, such as measurements of sound velocity in different material, attenuation coefficients, and directivity pattern of ultrasonic transducers. The objective of the proposed project is to implement the material developed at the Center for NDE (CNDE) at Iowa State University into a format suitable for undergraduate Applied Engineering Technology students and expand it to an Internet-based course composed of an introductory laboratory course similar to the one already offered but with a problem-based learning approach to NDT of materials. The developed course will be offered to AET students in Electrical, Mechanical, and Manufacturing concentration areas in accordance with Continuous Quality Improvement process. This laboratory course will introduce AET students to the engineering principles of ultrasound measurements by combining hands-on laboratory experience with lectures. More specifically, the students will learn the engineering and physical principles of measurements of sound velocity in different materials, attenuation coefficients, directivity pattern of ultrasonic transducers, and location and dimensions of heterogeneities in various materials, such as flaws, cavities, layers, and holes. Students will become familiar with calibration procedures of the industrial NDT equipment. The industrial case studies in laboratory environment will enhance the fundamentals taught in the classroom sessions.

Desired learning outcomes for students who complete this course are:

- Students will become familiar with basic ultrasonic instrumentation, gain hands-on experience with ultrasonic and electronic equipment, and be able to demonstrate the basic principles of ultrasound imaging.

- Students will be able to use an ultrasonic inspection system for quality control analysis of various materials incorporating the latest development in imaging software technology, ultrasonic instrumentation, and mechanical positioning hardware assemblies.

- Students will become familiar with the practical methods and techniques of NDE used in the industry and will improve their problem solving capabilities.

- Students will carry out laboratory procedures and techniques with guidance from an instructor, and describe the purpose of the procedures, techniques, and the results in their laboratory reports.

This course will be designed for undergraduates at the pre-junior or junior levels, but may also be taken by other undergraduate and graduate students who have fulfilled the necessary prerequisites and desire to obtain knowledge in the field of ultrasound NDT of materials. This 
approach will provide an excellent introduction to the manufacturing environment for the undergraduates, develop project leadership skills, and facilitate the development of teamwork that will allow the project to proceed without the constant supervision of the faculty advisor.

\section{LABORATORY PROCEDURES}

During the fall term of the 2007-2008 academic year, the developed three-credit course EET-203 (Nondestructive Evaluation of Materials) was offered to pre-junior AET students. Laboratory procedures were organized around current developments in the field of ultrasound NDT of materials. During the laboratory sessions, students were introduced to tools, methodologies, and techniques used by the NDT specialists in real-world applications. Students carried out experiments under the supervision of the instructor and described the results of the experiments in individual reports for each laboratory session. After completion of all laboratory sessions, each team was responsible for writing a final report that summarized the current state in the area, described the experimental techniques utilized, discussed the expected outcomes, provided data of the actual outcomes, and explained the reasons for the departures between the expected and the actual results. The teams analyzed the data, described conclusions, and suggested possible ways for improving the accuracy of their experiments. During the final presentation, the teams presented their findings to the class as a whole.

The following experiments were carried out during the laboratory sessions to introduce students to the fundamentals of ultrasound nondestructive testing:

- Measurements of the sound velocity in water

- Measurements of the sound velocity in other materials

- Directivity Pattern Measurements

- Measurements of the attenuation coefficient of the ultrasonic waves

In order to incorporate the NDT procedures, the laboratory was equipped by the portable Ultrasonic Flaw Detectors USN58L and USM35X (Fig. 1), which allows the following experiments to be implemented ${ }^{2}$ :

- Calibration of automatic flaw detectors

- Evaluation of homogeneity of various materials used in industrial applications

- Detection and localization of heterogeneities in the materials, such as flaws, cavities, layers, and holes $3,4,5,6$

- Measurement of the dimensions of various parts and components, where conventional methods (such as rulers and calipers) cannot be applied 

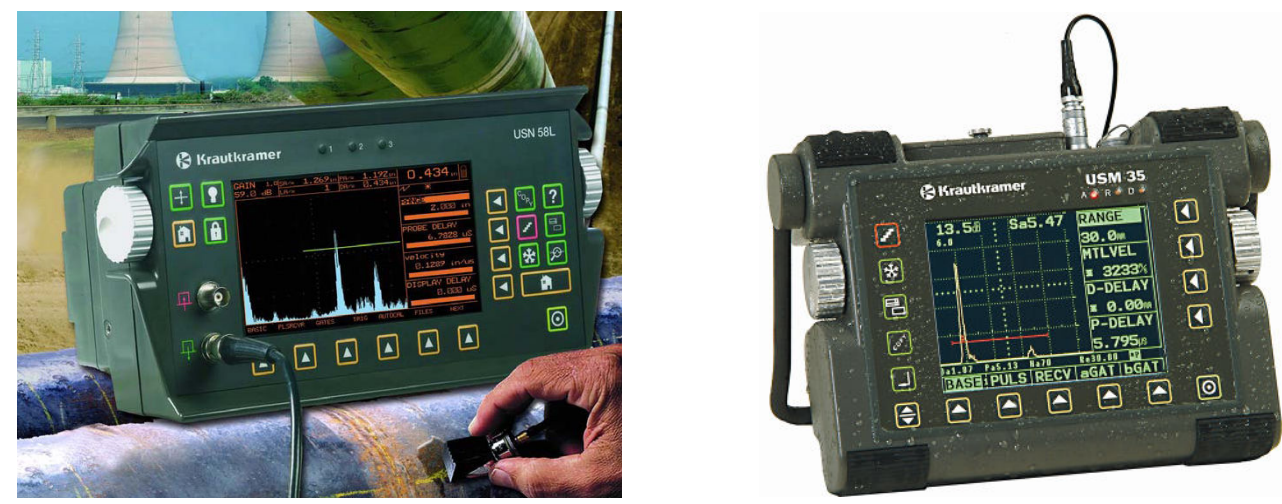

Fig. 1. Portable Ultrasonic Flaw Detectors USN58L (left) and USM 35X (right)

Specifically, the following experiments were carried out:

1. Calibration of the flaw detectors using the step calibration block and Straight-Beam single-element or dual-element probes utilizing the instruments' AUTO-CAL feature (Fig. 2). During the procedure, students set the display RANGE so that both reference calibration echoes from different material thicknesses are displayed on the screen (Fig. 3 and Fig. 4).

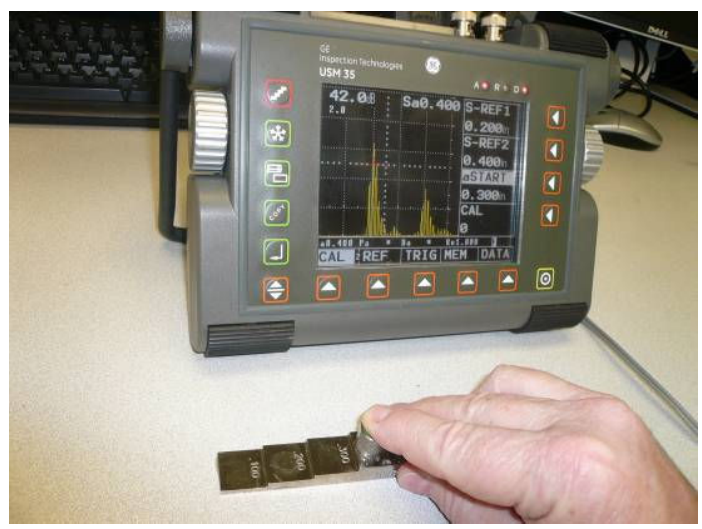

Fig. 2. Calibration of the flaw detectors using a straight-beam probe

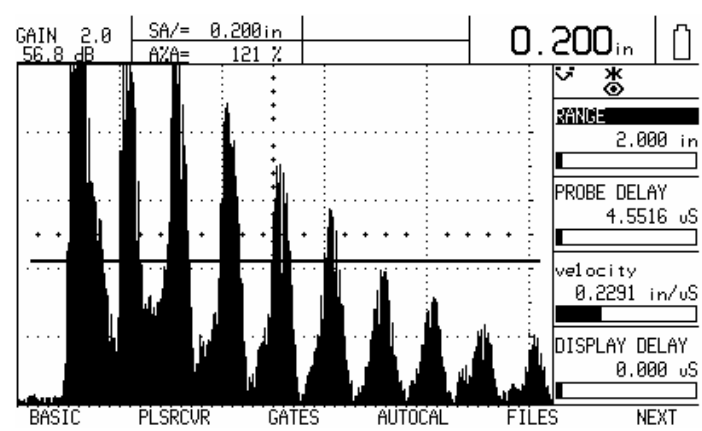

Fig. 3. First calibration echo

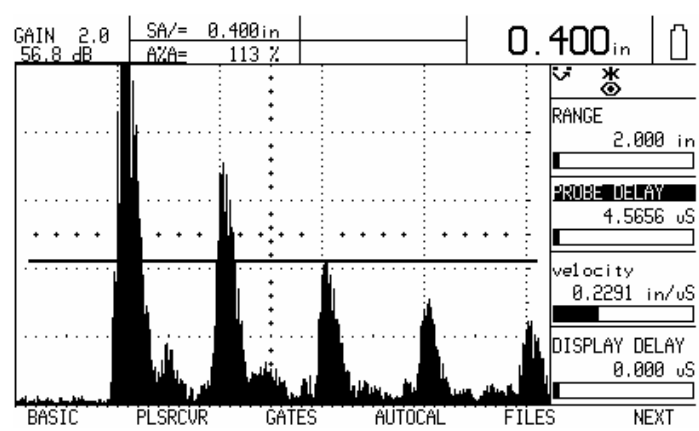

Fig. 4. Second calibration echo 
The correct calibration is confirmed by the message "CALIBRATION IS DONE." The flaw detector can now automatically determine the sound velocity of the material being used in this procedure and the probe delay.

2. Evaluation of the resolution of the system, i.e. the ability of the system to differentiate two or more discontinuities closely spaced in a lateral plane (Fig. 5). After the calibration of the instrument, the detection of discontinuities is carried out with the same instrument and the same probe.

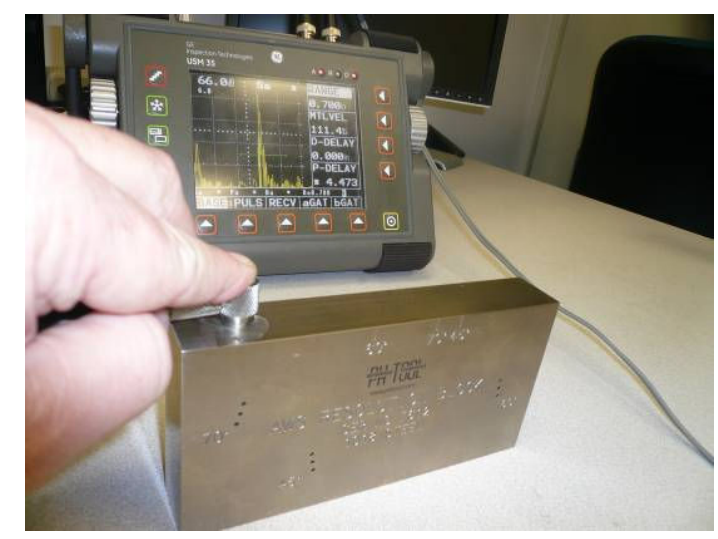

Fig. 5. Evaluation of the resolution of the system

3. Calibration procedure with the Angle-Beam probe for Wedge Angle Verification, Sound Path Distance Calibration, and Flaw Sensitivity Calibration using an IIW (International Institute of Welding) type 1 calibration block is presented below (Fig. 6).

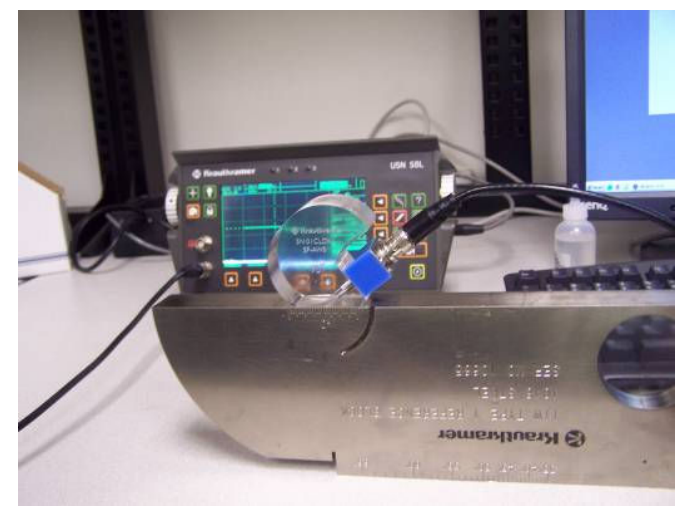

Fig. 6. IIW calibration block

The results of the calibration procedure using an angle-beam probe are presented in Fig. 7. 




Fig.7. Results of the calibration procedure using an angle-beam probe

After calibration of the instrument, students place the transducer at various positions on the IIW block to display reflections from sound path distances and sensitivity relationships to side drilled holes.

\section{REMOTE CONTROL OF THE NDT EQUIPMENT}

One of the main objectives of the project is to develop a videoconference teaching NDT facility, which will provide greater program delivery flexibility and offer non-traditional educational segments that expand student's horizons. ${ }^{7,8,9}$ This facility will allow all AET students at Drexel, as well as students at remote locations, to be involved in the same educational and training process in NDT. By expanding training opportunities to students who might not otherwise take advantage of them, due to distance and time, this facility helps reduce the shortage of trained specialists in NDT field. Key factors in the development process include creation of the educational laboratory that can significantly contribute to the development of technologically literate students and workforce that will be in great demand not only in the tri-state area but also nationwide. The fully-interactive videoconference teaching course in NDT was designed for undergraduate AET students and may also be taken by other undergraduate/graduate students at Drexel or by the students of other universities and community colleges who have fulfilled the necessary prerequisites and desire to pursue a BS degree in AET or obtain training in NDT. This approach will facilitate the development of teamwork that will allow the project/laboratory to proceed without the constant supervision of the faculty advisor. ${ }^{10}$ The inter-institutional class sessions will be carried out utilizing Internet2-based access to the equipment of Drexel's NDT laboratory for other universities and community colleges. Students involved in the "live" interaction with other participants will share in small group discussions, share documents, collaborate, and fully engage in the videoconferencing experience. The implications of fullyinteractive videoconference teaching are far-reaching as it relates to distance delivery of realtime interactive instruction between any remote sites subscribing to Internet 2 services using Internet Protocol (IP) networks. The state of the art NDT facility is also designed to serve working individuals interested in improving their skills in NDT, as well as those seeking knowledge for professional advancement.

The backbone of the IP-based videoconferencing consists of Polycom VSX7000 system. The local site with portable ultrasonic flaw detectors utilizes Sony HDR-SR5 camcorder for capture and recording of the experiments and several LCD or Plasma monitors for visual display of the 
activities. GE Inspection Technologies' UltraDoc software allows for control and data transfer to and from the portable ultrasonic flaw detectors. UltraVNC (Virtual Network Computing) software enables remote control and data transfer from the local computer connected to the Flaw Detectors and the camcorder simultaneously. Utilizing UltraVNC and UltraDoc control function and commands, one can remotely control and change any setting of the Flaw Detectors, such as calibration of flaw detectors and evaluation of the test objects.

This configuration of equipment and software packages allow students at the remote site for participation in the laboratory activities. Technician's or teaching assistant's presence at the local site is required for initial set-up of the videoconferencing and NDT equipment and handling of the transducers. The calibration of the NDT equipment and testing procedures can be completely controlled from the remote site. Results of the calibration and testing could be saved from both local and remote computers. The block diagram of the remote NDT procedure is presented in Fig. 8.

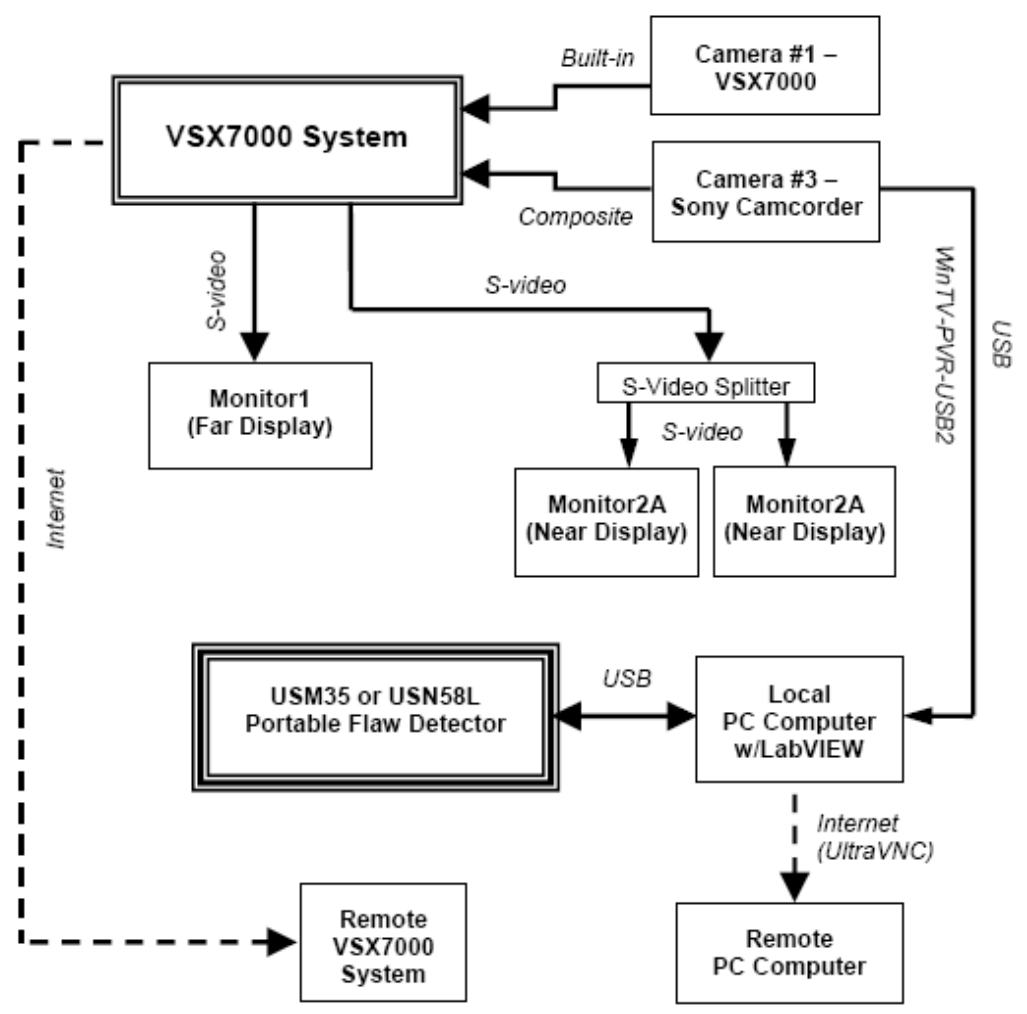

Fig. 8. Block diagram of the remote NDT procedure

\section{PROJECT ASSESSMENT AND EVALUATION}

Evaluation of the project and data collection will begin soon after the start of the project.

Students will be administered a pre-test, which assesses the entering knowledge requirements for the course. For example, their knowledge of appropriate algebra, trigonometry, and vibrations and sound waves will be evaluated. Based on the results of the test, students will be divided by groups according to the think-share-report-learn (TSRL) process, which will involve student peer 
coaching to help each other during the laboratory procedures. The sophistication of this approach to teaching the problem-based hands-on learning model within a virtual environment of a stateof-the-art laboratory for NDE purposes will be evaluated using both formative and summative evaluation. A formative evaluation will assess initial and ongoing project activities based on the students' laboratory reports, which are required within seven days after each laboratory session. A summative evaluation will assess the quality and impact of an implemented project based on the students' final presentations, including corrections of the collected results and conclusions. Industrial evaluators will be involved in both formative and summative evaluations. After completion of the course, students will complete a course evaluation, including the following questions according to a Likert-type scale, which assesses the students' perception of their confidence, knowledge, and competence. Below are Likert-type items.

\begin{tabular}{|c|c|c|c|}
\hline \multicolumn{3}{|c|}{ a) As an AET engineer, do you feel confident in NDE?* } \\
\hline Not at all confident & A little confident & Pretty confident & Very confident \\
\hline \multirow{2}{|c|}{ b) As an AET engineer, do you feel knowledgeable in NDE? } \\
\hline $\begin{array}{c}\text { Not at all } \\
\text { knowledgeable }\end{array}$ & A little knowledgeable & Pretty knowledgeable & Very knowledgeable \\
\hline \multirow{2}{*}{ c) As an AET engineer, do you feel competent in NDE? } & \\
\hline & & & Pery competent \\
\hline Not at all competent & A little competent & Pretty competent & \\
\hline &
\end{tabular}

* Put an "X" on the line under the correct answer that shows how you feel about each statement.

The student course evaluations and instructor course evaluation will be assessed by the independent evaluator for purposes of course modifications, where appropriate. 


\section{SUMMARY}

During the fall term of the 2007-2008 academic year, the course EET-203 (Nondestructive Evaluation of Materials) was offered to pre-junior AET students. This hands-on lecture/laboratory course consists of two parts: the first part with an emphasis on the foundations of NDE, and the second part where nondestructive evaluation techniques of parts and materials is presented and applied through real-life problems. Specifically, the students learn calibration techniques of the NDT equipment and testing procedures regarding detection and evaluation of various discontinuities in the materials, such as holes, cracks, cavities, and flaws. Both calibration and testing procedures can be carried out locally where NDT equipment is installed or remotely using two-way fully-interactive facility. This approach can be used for education, training, and real-life industrial applications. Fully-interactive videoconference teaching will create infrastructure for remote access to high-end equipment via the Internet2, as well as provide experience in effective delivery of education through this modality. It will also create a model for incorporating leading edge concepts and technology in AET education into undergraduate research that can be utilized at other institutions and in other disciplines.

The success of the project will be evaluated by the unbiased evaluators using both formative and summative evaluation based on the students' quizzes, laboratory reports, and final presentations, including corrections of the collected results and conclusions.

\section{REFERENCES}

1. V. Genis, H. Sosa, \& E. Radulescu. Ultrasound Measurements and Nondestructive Testing Educational Laboratory. Proceedings of the ASEE Conference, pp. 1-9, June 2004.

2. Dong Fei, David Hsu, and Mark Warchol. Journal of NDE, Vol. 20, No. 3, 2002, pp. 95-112.

3. Nondestructive Testing. Volume I - Basic Principles. General Dynamics. ASNT Publication, 2002.

4. Nondestructive Testing. Volume II - Equipment. General Dynamics. ASNT Publication, 2002.

5. Nondestructive Testing. Volume III - Applications. General Dynamics. ASNT Publication, 2002.

6. Paul Mix. Introduction to Nondestructive Testing. A Training Guide. A John Wiley \& Sons, 2005.

7. Aktan, B., Bohus, C.A., Crowl, L.A. \& Shor, M.H., 1996, Distance learning applied to control engineering laboratories, IEEE Transactions on Education, Vol. 39, No. 3, pp. 320-326.

8. Casini, M., Prattichizzo, D. \& Vicino, A., 2003, The automatic control telelab: a user-friendly interface for distance learning, IEEE Transactions on Education, Vol. 46, No. 2, pp. 252-257.

9. Hong Shen, Zheng Xu, Dalager, B., Kristiansen, V., Strom, O., Shur, M.S., Fjeldly, T.A., Jian-Qiang Lu \& Ytterdal, T., 1999, Conducting laboratory experiments over the Internet, IEEE Transactions on Education, Vol. 42, No. 3, pp. 180-185.

10. V. Genis, A. Daneshpooy, and C. O'Shaughnessy. Development of Videoconference Teaching for Applied Engineering Technology Students. Proceedings of the ASEE Mid-Atlantic Conference, pp. 1-6, April 2005. 